\title{
Clock dial integrated positioning combined with single utility port video-assisted thoracoscopic surgery: a new localization method for lung tumors
}

\author{
Chao Zhou ${ }^{1}$, Xinming Li $^{2}$, Wentao Li $^{1}$, Jun Qian ${ }^{2}$ \\ ${ }^{1}$ Department of Thoracic Surgery, Shanghai Chest Hospital, Shanghai Jiao Tong University, Shanghai, China; ${ }^{2}$ Department of Cardiothoracic \\ Surgery, Dehong People's Hospital, Affiliated Dehong People's Hospital of Kunming Medical University, Dehong, China \\ Contributions: (I) Conception and design: J Qian, W Li; (II) Administrative support: C Zhou, J Qian, X Li; (III) Provision of study materials or \\ patients: C Zhou, J Qian, W Li; (IV) Collection and assembly of data: C Zhou, J Qian, X Li; (V) Data analysis and interpretation: C Zhou, J Qian; (VI) \\ Manuscript writing: All authors; (VII) Final approval of manuscript: All authors. \\ Correspondence to: Jun Qian. Department of Cardiothoracic Surgery, Affiliated Dehong People's Hospital of Kunming Medical University, Dehong, \\ China. Email: 175382204@qq.com; Wentao Li. Department of Thoracic Surgery, Shanghai Chest Hospital, Shanghai Jiao Tong University, \\ Shanghai, China. Email: Liwentootoo@163.com.
}

Background: Preoperative localization of lung tumor mainly consisted of two methods: CT-guided percutaneous localization and electromagnetic navigation bronchoscopy-guided localization. However, these invasive methods could result in serious complications. In order to avoid the adverse effects of preoperative invasive localization, we propose a method of intraoperative noninvasive localization for lung tumors: clock dial integrated positioning (CDIP).

Methods: To retrospectively analyze the clinic data about the application of CDIP for 127 lung tumour patients in single utility port video-assisted thoracoscopic surgery (SUPVATS) between June 2017 and October 2017.

Results: One hundred and twenty-four cases (97.64\%) underwent thoracoscopic surgery, which including 14 lobectomy, 107 partial resection, 2 lobectomy plus partial resection and 1 left pneumonectomy. Three cases $(2.36 \%)$ underwent thoracoscopic biopsy. The mean operation time and intraoperative bleeding were $47.9 \pm 22.1 \mathrm{~min}$ and $70.1 \pm 40.3 \mathrm{~mL}$, respectively. The mean postoperative hospital stay and chest drain duration were $3.9 \pm 2.2$ and $3.6 \pm 1.8$ days, respectively. There were 118 cases of malignant tumors, including adenocarcinoma $(n=101)$, squamous cell carcinoma $(n=9)$, large cell carcinoma $(n=2)$, small cell lung carcinoma $(n=3)$, and metastatic lung carcinoma $(n=3)$. The remaining nine cases were benign tumors, including granuloma $(n=3)$, intrapulmonary lymph node $(n=2)$, sclerosing hemangioma $(n=2)$, and hamartoma $(n=2)$. The incidence of postoperative complications was $10.2 \%$. There was no mortality, secondary operation, or conversion to open procedure due to massive intraoperative bleeding.

Conclusions: CDIP combined with SUPVATS is a safe, feasible, and effective method for the localization of lung tumors. This novel method can provide a reliable alternative technique when the marker is dislocated.

Keywords: Clock dial integrated positioning (CDIP); single utility port video-assisted thoracoscopic surgery (SUPVATS); lung tumors; non-small cell lung cancer (NSCLC)

Submitted Oct 20, 2020. Accepted for publication Jan 02, 2021.

doi: $10.21037 /$ jtd-20-3312

View this article at: http://dx.doi.org/10.21037/jtd-20-3312 


\section{Introduction}

With advances in the global economy and, the number of people with lung tumors has dramatically increased worldwide $(1,2)$. An increasing number of patients with lung tumors and ground glass nodules (GGNs) have been identified through computed tomography (CT), with the majority of these cases being early-stage lung cancers. Video-assisted thoracoscopic surgery (VATS) with systemic lymph node dissection is considered as the standard treatment for early non-small cell lung cancer (NSCLC) $(3,4)$. Nowadays, preoperative invasive localization of lung tumor mainly consists of two methods: CTguided localization (5-9) and electromagnetic navigation bronchoscopy (ENB)-guided localization (10-12). However, these methods have some disadvantages such as the lack of conditions in some backward areas, increased preoperative trauma and anxiety, etc. As well as some serious complications such as intrapulmonary focal hemorrhage, pneumothorax, chest pain requiring analgesia, hemosputum and so on (5-7).

The purpose of this study was to introduce a new intraoperative noninvasive localization method to locate lung tumors, which showed satisfactory results. This is the first report about the application of clock dial integrated positioning (CDIP) combined with single utility port VATS (SUPVATS) in the treatment of lung tumors. We present the following article in accordance with the STROBE reporting checklist (available at http://dx.doi.org/10.21037/ jtd-20-3312).

\section{Methods}

\section{Patient selection}

Between June 2017 and October 2017, a total of 127 patients with lung tumors underwent CDIP combined with SUPVATS at the Shanghai Chest Hospital of Shanghai Jiao Tong University. The patients' characteristics are listed in Table 1. Clinical staging of NSCLC was based on the Union for International Cancer Control (UICC) staging (8th edition) (13). All procedures performed in this study involving human participants were in accordance with the Declaration of Helsinki (as revised in 2013). This study was approved by the Institutional Review Board and Ethics Committee of the Shanghai Chest Hospital of Shanghai Jiao Tong University. All patients underwent preoperative routine echocardiography, abdominal ultrasonography, pulmonary function measurement, blood examination, chest CT scan, bone scintigraphy scan, and brain magnetic resonance imaging (MRI) to exclude extra-thoracic spread of the disease. The detailed medical history of all patients was documented, and all patients granted their informed consent at least one day before surgery, following conscientious explanation of the procedure and the goals of the study.

The inclusion criteria (14) were as follows: (I) initial stages of NSCLC $\mathrm{T}_{1} \mathrm{~N}_{0} \mathrm{M}_{0}$ to T1N1M0, or isolated single N2 lymphadenectasis of phase IIIA; (II) for smokers, the patient ceased smoking for at least 2 weeks prior to the operation; (III) preoperative lung function: forced expiratory volume (FEV1)\% and maximal voluntary ventilation (MVV) $\%$ of predicted $>50 \%, \mathrm{FEV} 1>1 \mathrm{~L}$; and (IV) no history of thoracic surgery or severe chest wall deformity. The exclusion criteria (14) were as follows: (I) tumor invasion of the large vessels of the mediastinum or important nerves such as the recurrent laryngeal nerve; (II) tumor invasion of large chest walls requiring reconstruction; (III) multi-station lymphatic metastasis; (IV) tumor invasion of the protuberance or trachea; (V) mediastinal lymphoid tuberculosis; (VI) severe chest wall deformity; (VII) upper airway and maxillofacial injury or deformity; and (VIII) lung, pleural, or heart disease.

\section{Surgical procedure}

\section{Localization of lung tumors using CDIP}

The CDIP method was applied to achieve intraoperative localization, based on the preoperative CT scans. The right chest with the Azygos vein arch and lower pulmonary vein, and the left chest with the aortic arch and the lower pulmonary vein, were tagged, and the lung was divided into the upper, middle, and lower parts according to the anatomical structure of the chest (Figure 1). On the right side of the tumors, the CT was rotated 90 degrees clockwise (Figure 2A,B). On the left side of the tumors, the CT was rotated 90 degrees counterclockwise (Figure 2C,D). CDIP was used to locate the midaxillary line at the 12 o'clock position, and the upper, middle, and lower parts of the lung were preoperatively identified to accurately locate the tumors. The vertical distance and clock dial points were used to locate the lung tumor.

The specific measures were as follows: firstly, based on the CT scan, we calculated the vertical distance of the tumors (according to the azygos vein arch, aortic arch, and lower pulmonary vein), and determined the part in which the tumor was located (Figure 1). Secondly, we calculated 
the clock dial position of the tumors according to the above method (Figure 2). Thirdly, we used an electrotome to mark the tumor site in the lung. Lastly, the operations were centered in that region, and thoracoscopic surgery was performed.

\section{Surgical technique and localization procedure}

Under general anesthesia with a double-lumen tube in the

Table 1 Patient characteristics $(\mathrm{N}=127)$

\begin{tabular}{lc}
\hline Characteristic & $\mathrm{N}$ \\
\hline Age (years, mean \pm SD) & $57.2 \pm 10.6$ \\
Gender & 49 \\
Male & 78 \\
Female & \\
Localization of lung tumors & $32 / 3 / 27$ \\
Right upper lobe/right middle lobe/right lower lobe & $35 / 18$ \\
Left upper lobe/left lower lobe & 5 \\
Right upper lobe and lower lobe & 2 \\
Right upper lobe and middle lobe & 1 \\
Right lower lobe and middle lobe & 4 \\
Left upper lobe and lower lobe & \\
Tumor sizes (mm) & 97 \\
$<10$ & 17 \\
$10-20$ & 13 \\
$>20$ & \\
\hline
\end{tabular}
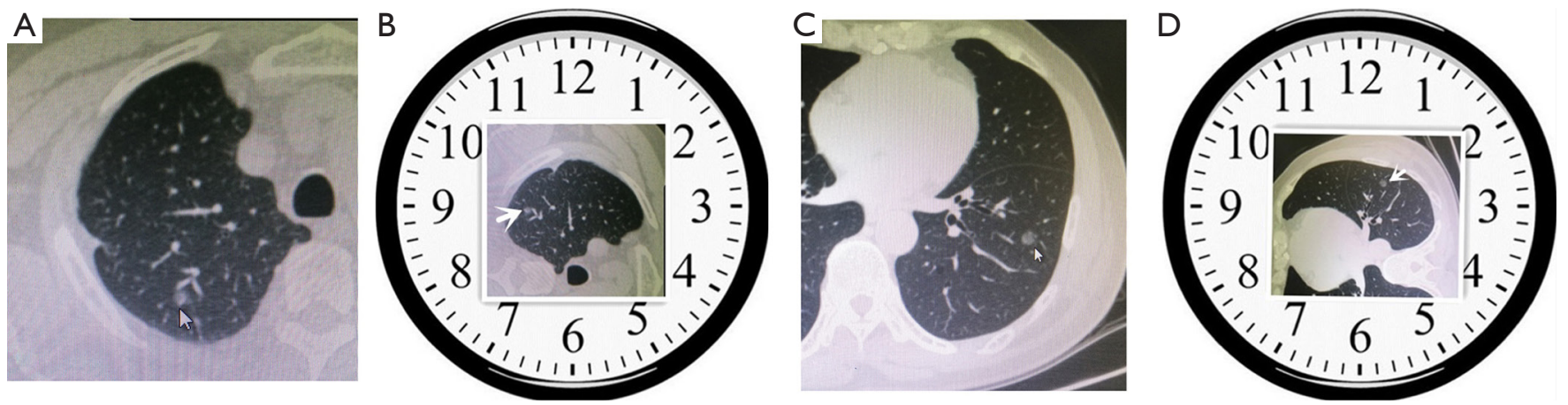

Figure 2 The method of clock dial integrated positioning (CDIP). (A) The clock dial integrated positioning shown on the right side of the tumors before surgery; (B) clockwise rotation of 90 degrees, the tumor is located at the 9 o'clock positionand above the azygos arch is belongs to the upper part; (C) on the left side of the tumor before surgery; (D) counterclockwise rotation of 90 degrees, the tumor is located at the 1 o'clock position and is at the level of the lower pulmonary vein. Arrows indicate changes in the chest. 

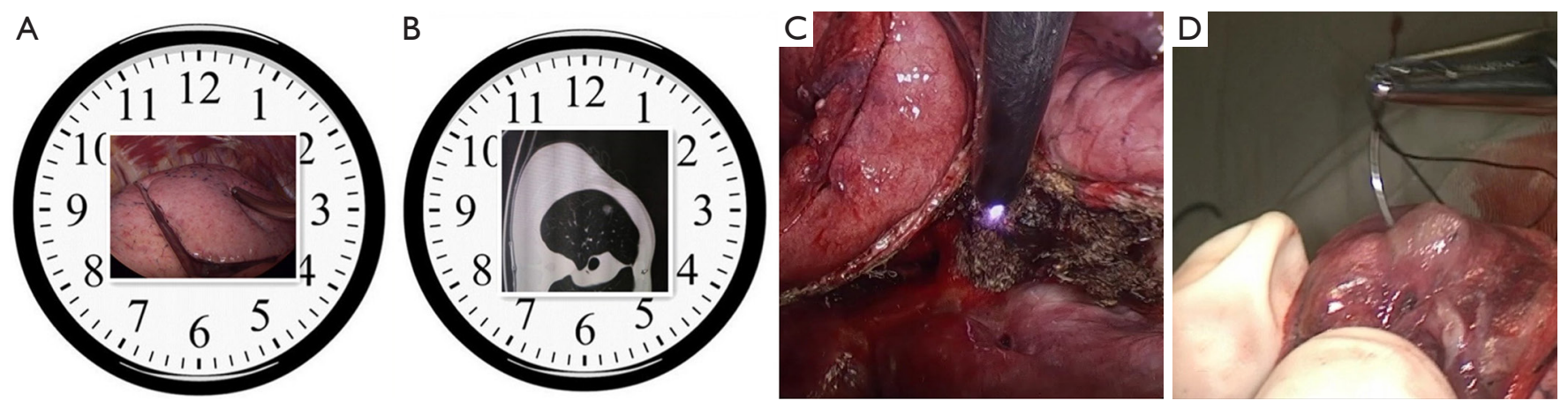

Figure 3 The CDIP located the right upper lung tumor intraoperatively. (A) The CDIP shown on the right side of the tumor is located at the 1 o'clock position and above the azygos arch in the upper part before surgery; (B) the area is above the azygos arch that is located at the 1 o'clock position during the surgery; (C) SUPVATS excision of the tumors; (D) tumors were located in this area. CDIP, clock dial integrated positioning; SUPVATS, single utility port video-assisted thoracoscopic surgery.

on the preoperative CT scan (Figure 3). If the tumor was difficult to locate, the anesthesiologist would drum the lung so that we could accurately determine the location of the tumor in the expanded state. SUPVATS excision of the tumors included lobectomy, segmentectomy, wedge resection, and mediastinal lymph node dissection or sampling. If 13 th or 11 th lymph node metastasis was confirmed, we extended the resection or performed lobectomy, as required. In general, most tumors were resected in the first thoracoscopic surgery, while other tumors were resected by extended resection or lobectomy. When pleural irrigation and lung expansion showed no active hemorrhage or air leakage, a chest tube was placed directly through the observation port (14). The chest tube was removed $(14,15)$ when: (I) the amount of drainage was $<100 \mathrm{~mL} /$ day; (II) the lung was fully expanded without pleural effusion on chest radiography; and (III) there was no air leakage through the chest tube.

\section{Data collection and follow-up}

The surgical type, operation time, intraoperative bleeding, postoperative hospital stay, chest drain duration, postoperative pathological TNM stage of NSCLC, and complications were recorded. Among the 127 patients, 116 cases $(91.34 \%)$ were followed-up, while 11 cases $(8.66 \%)$ were lost to follow-up.

\section{Statistical analysis}

Clinicopathologic data was analyzed using the SPSS 22.0 software package (SPSS Inc., Chicago, IL). Continuous variables were expressed as mean \pm standard deviation (SD). Categoric variables were expressed as count and percentages.

\section{Results}

\section{Surgical methods and related data}

In this study, only 3 cases (2.36\%) underwent thoracoscopic biopsy due to incomplete resection, while the remaining 124 cases $(97.64 \%)$ underwent thoracoscopic surgery. This included 14 cases of lobectomy, 107 cases of partial resection (including segmental or wedge resection), 2 cases of lobectomy and partial resection, 1 case of left total pneumonectomy, and 3 cases of thoracoscopic biopsy. In two patients, the lung tumors could not be found during the first thoracoscopic surgery, but were subsequently removed by lobectomy and extended resection, respectively.

\section{General postoperative and pathology data}

The mean operation time and intraoperative bleeding were $47.9 \pm 22.1 \mathrm{~min}$ and $70.1 \pm 40.3 \mathrm{~mL}$, respectively. The mean postoperative hospital stay and chest drain duration were $3.9 \pm 2.2$ and $3.6 \pm 1.8$ days, respectively. There were 118 cases of malignant tumors, including 101 cases of adenocarcinoma, 9 cases of squamous cell carcinoma, 2 cases of large cell carcinoma, 3 cases of small cell lung carcinoma, and 3 cases of metastatic lung carcinoma. The remaining 9 cases were benign tumors, including 3 cases of granuloma, 2 cases of intrapulmonary lymph node, 2 
Table 2 Clinicopathologic characteristics of the patients ( $N=127)$

\begin{tabular}{|c|c|}
\hline Clinicopathologic characteristics & $\mathrm{N}$ \\
\hline \multicolumn{2}{|l|}{ Surgical type } \\
\hline Lobectomy & 14 \\
\hline $\begin{array}{l}\text { Partial resection } \\
\text { (including segmental or wedge resection) }\end{array}$ & 107 \\
\hline Lobectomy and partial resection & 2 \\
\hline Thoracoscopic biopsy & 3 \\
\hline Left total pneumonectomy & 1 \\
\hline Operation time, mean $\pm \mathrm{SD}(\mathrm{min})$ & $47.9 \pm 22.1$ \\
\hline Intraoperative bleeding, mean $\pm \mathrm{SD}(\mathrm{mL})$ & $70.1 \pm 40.3$ \\
\hline Postoperative hospital stay, mean \pm SD (days) & $3.9 \pm 2.2$ \\
\hline Chest drain duration, mean $\pm S D$ (days) & $3.6 \pm 1.8$ \\
\hline \multicolumn{2}{|l|}{ Postoperative pathology } \\
\hline Malignant tumor & 118 \\
\hline Adenocarcinoma & 101 \\
\hline Squamous cell carcinoma & 9 \\
\hline Large cell carcinoma & 2 \\
\hline Small cell lung carcinoma & 3 \\
\hline Metastatic lung carcinoma & 3 \\
\hline Benign tumor & 9 \\
\hline Granuloma & 3 \\
\hline Intrapulmonary lymph node & 2 \\
\hline Sclerosing hemangioma & 2 \\
\hline Hamartoma & 2 \\
\hline \multicolumn{2}{|l|}{ TNM stage } \\
\hline$I_{1}$ & 83 \\
\hline $\mathrm{IA}_{2}$ & 10 \\
\hline $\mathrm{IA}_{3}$ & 7 \\
\hline IB & 2 \\
\hline$\| A$ & 2 \\
\hline IIB & 2 \\
\hline IIIA & 6 \\
\hline
\end{tabular}

cases of sclerosing hemangioma, and 2 cases of hamartoma. According to the clinical staging of NSCLC based on the UICC staging (8th edition) (13), 83 cases were stage IA1, 10 cases were stage IA2, 7 cases were stage IA3, 2 cases were stage IB, 2 cases were stage IIA, 2 cases were stage IIB, and
Table 3 Postoperative complications of patients $(\mathrm{N}=13)$

\begin{tabular}{ll}
\hline Complications & $\mathrm{N}(\%)$ \\
\hline Postoperative pneumonia & $7(5.51)$ \\
Atelectasis & $2(1.57)$ \\
Prolonged air leakage (>5 days) & $2(1.57)$ \\
Hypoxia & $1(0.79)$ \\
Arrhythmia & $1(0.79)$ \\
\hline
\end{tabular}

6 cases were stage IIIA. The clinical characteristics of the 127 patients are shown in Table 2.

\section{Data of postoperative complications and follow-up}

All patients were hospitalized. The incidence of postoperative complications was $10.2 \%$; postoperative pneumonia was observed in 7 cases $(5.51 \%)$, atelectasis in 2 cases $(1.57 \%)$, prolonged air leakage ( $>5$ days) in 2 cases $(1.57 \%)$, hypoxia in 1 case $(0.79 \%)$, and arrhythmia in 1 case $(0.79 \%)$. The postoperative complications are shown in Table 3. All patients were successfully discharged after surgery, and there was no mortality, secondary operation, or conversion to open procedure due to massive intraoperative bleeding. The 116 patients were followed-up for 6-12 months by outpatient (103 cases, $88.79 \%$ ), telephone (10 cases, $8.62 \%$ ), and e-mail (3 cases, $2.59 \%$ ), respectively. The mean duration of follow-up was $9.1 \pm 1.8$ months. Two cases developed pulmonary metastasis at 6 months and bone metastases at 11 months after surgery, respectively. No deaths occurred during the follow-up period.

\section{Discussion}

The department of thoracic surgery at the Shanghai Chest Hospital of Shanghai Jiao Tong University is the largest thoracic centre in China, and deals with the complete spectrum of diseases and disorders. We have performed more than 10,000 thoracic surgeries in 2019; thoracoscopic surgery accounted for $>80 \%$ of cases, and robotic surgery accounted for $>3 \%$ of cases. There are numerous earlystage lung cancer patients at our centre, and the localization of lung tumors is a primary concern. Various methods are used in our clinic for the localization of lung tumors, including the hook wire, vital dye, transbronchial, and CDIP. At present, local resection has become an option for early-stage lung cancer, which requires us to be able 
to pinpoint the location of the lung tumor, so that we can ensure cutting margin for surgery in order to preserve more normal tissue.

In this study, only 3 cases $(2.36 \%)$ underwent thoracoscopic biopsy due to incomplete resection, while the remaining 124 cases $(97.64 \%)$ underwent thoracoscopic surgery. In two patients, the lung tumors could not be found during the first thoracoscopic surgery, but were subsequently removed by lobectomy and extended resection, respectively. Tables 2 and 3 demonstrate satisfactory efficacy and low postoperative complications in all patients. All patients were successfully discharged after surgery, and there was no mortality, secondary operation, or conversion to open procedure due to massive intraoperative bleeding. During the follow-up (after stenting), two cases developed pulmonary metastasis at 6 months and bone metastases at 11 months after surgery, respectively. No deaths occurred during the follow-up period.

The ability of our team to achieve these positive outcomes is attributable to several factors. Firstly, Professor $\mathrm{Li}$ has extensive surgical experience, and has performed several thousand thoracic surgeries. A previous study (16) showed that a low complication rate was achieved if the surgery was performed by experienced surgeons after proper training. Secondly, we have implemented complete perioperative education and guidance for patients and their families. We urge patients to give up smoking, strengthen nutritional support, improve perioperative exercise capacity, and actively cough and expectorate during the perioperative period, which can promote wound healing, reduce thrombotic disease, and reduce postoperative pneumonia and atelectasis. Several studies have reported the effectiveness of these measures during the perioperative period of lung cancer surgery (17-19). Leone et al. (17) found that tobacco abstinence contributed to improved patient-related outcomes at various phases of lung cancer management. The European Society for Clinical Nutrition and Metabolism (ESPEN) guidelines (18) highlight the nutritional aspects of the enhanced recovery after surgery (ERAS) concept and the special nutritional needs of patients undergoing major surgery. Another study (19) found that rehabilitation programs and exercise-based interventions can improve exercise capacity and lung cancer outcomes. Exercise capacity is prognostic and predictive of outcomes in lung cancer, and it is critical to risk-stratify patients undergoing evaluation for lung cancer resection. Thirdly, among these patients, 100 cases $(78.74 \%)$ were IA stage NSCLC, while 107 cases (84.25\%) underwent partial resection (including segmental and wedge resection), which requires less resection and involves less surgical trauma. A previous study (20) advocated sublobar resection for NSCLC patients with compromised cardiopulmonary reserve, as well as for selected patients with early-stage disease. Therefore, we also performed partial resection for early-stage NSCLC or patients with poor pulmonary function. Moreover, we routinely conduct intraoperative pathological examination of the 11th group of lymph nodes and margins, as well as its metastasis, to determine if we need to expand surgical resection or perform lobectomy.

In clinical practice, we found that when the tumor diameter was $>5 \mathrm{~mm}$, it could be easily detected by preoperative high-resolution CT. According to the 8th edition staging (13) of NSCLC, a tumor diameter of $<10 \mathrm{~mm}$ is T1a, and most cases are adenocarcinoma in situ and minimally invasive adenocarcinoma or lepidic patternpredominant adenocarcinoma; N2 lymph node metastasis rarely occurs in these types (21). When the lung tumor diameter is $<5 \mathrm{~mm}$, it is very difficult to locate, even under preoperative positioning in surgery, and so the surgical treatment of this kind of tumor is not recommended. However, when the tumor diameter is $5-10 \mathrm{~mm}$, can accurately resect the tumors without changing the clinical stage of NSCLC. We found that the 100 patients (78.74\%) with IA stage NSCLC had good prognosis during follow-up.

Doctor Qian has worked under Professor Li for 1 year at the Shanghai Chest Hospital, and has completed $>100$ lung tumor surgeries in his unit using CDIP. Compared to the preoperative invasive lung tumor positioning method, we believe that this method offers the following advantages. Firstly, it does not require expensive medical equipment and complex preoperative invasive localization, which many grassroots hospitals in China cannot implement due to economic and technical factors limitations and so on. Secondly, during the perioperative period, invasive methods can lead to some severe complications (5-7,22-24); most commonly, intrapulmonary focal hemorrhage (54.2\%) (23), pneumothorax $(7.5-49 \%)(5,6,22,23)$, chest pain requiring analgesia (9\%) (5), hemosputum $(2.6-6 \%)(5,22)$, dislocation of the marker $(3.1-7.5 \%)(6,24)$, hemopneumothorax $(0.6 \%)$ (5), and systemic air embolism (0.24\%) (22). However, CDIP is used to locate the tumor during surgery, which can avoid the complications associated with invasive preoperative positioning. Furthermore, some patients have co-morbidities, including hypertension, diabetes, coronary heart disease, arrhythmia, cerebral infarction, etc., and preoperative invasive localization is also considered 
to be a high-risk operation in elderly patients. Moreover, preoperative invasive localization cannot be performed in some patients because of fear or physical condition, and thus, CDIP may benefit such patients. Thirdly, this approach can provide a reliable alternative when the marker is dislocated.

However, there are some disadvantages to CDIP. Firstly, the vertical distance and the clock dial position of tumors needs to be calculated according to the thoracic anatomy, which requires extensive clinical experience and good stereoscopic geometric imaging. Moreover, superficial lung tumors are relatively easy to locate, while deep lung tumor localization is extremely risk using this approach.

\section{Conclusions}

CDIP combined with SUPVATS is a safe, feasible, and effective method for the localization of lung tumors. This novel method can provide a reliable alternative technique for cases in which the marker is dislocated.

\section{Acknowledgments}

This project was supported by Yunnan Provincial Department of Science and Technology - Kunming Medical University Joint Special Project in 2019 under Grant (Detection of EGFR gene mutation and its clinical significance in non-small cell lung cancer in Dehong minority areas).

Funding: The research was supported by the joint project of Yunnan Provincial Department of Science and Technology and Kunming Medical University, China [2019FE001(281)], and the scientific research project of Shanghai Municipal Commission of Health and Family Planning (20164Y0211).

\section{Footnote}

Reporting Checklist: The authors have completed the STROBE reporting checklist. Available at http://dx.doi. org/10.21037/jtd-20-3312

Data Sharing Statement: Available at http://dx.doi. org/10.21037/jtd-20-3312

Conflicts of Interest: All authors have completed the ICMJE uniform disclosure form (available at http://dx.doi. org/10.21037/jtd-20-3312). The authors have no conflicts of interest to declare.

Ethical Statement: The authors are accountable for all aspects of the work in ensuring that questions related to the accuracy or integrity of any part of the work are appropriately investigated and resolved. All procedures performed in this study involving human participants were in accordance with the Declaration of Helsinki (as revised in 2013). This study was approved by the Institutional Review Board and Ethics Committee of the Shanghai Chest Hospital of Shanghai Jiao Tong University. All patients granted their informed consent at least one day before surgery, following conscientious explanation of the procedure and the goals of the study.

Open Access Statement: This is an Open Access article distributed in accordance with the Creative Commons Attribution-NonCommercial-NoDerivs 4.0 International License (CC BY-NC-ND 4.0), which permits the noncommercial replication and distribution of the article with the strict proviso that no changes or edits are made and the original work is properly cited (including links to both the formal publication through the relevant DOI and the license). See: https://creativecommons.org/licenses/by-nc-nd/4.0/.

\section{References}

1. Siegel RL, Miller KD, Jemal A. Cancer statistics, 2018. CA Cancer J Clin 2018;68:7-30.

2. Koike T, Togashi K, Shirato T, et al. Limited resection for noninvasive bronchioloalveolar carcinoma diagnosed by intraoperative pathologic examination. Ann Thorac Surg 2009;88:1106-11.

3. Cox ML, Yang CJ, Speicher PJ, et al. The Role of Extent of Surgical Resection and Lymph Node Assessment for Clinical Stage I Pulmonary Lepidic Adenocarcinoma: An Analysis of 1991 Patients. J Thorac Oncol 2017;12:689-96.

4. Wang GS, Wang Z, Wang J, et al. Uniportal complete video-assisted thoracoscopic lobectomy with systematic lymphadenectomy. J Thorac Dis 2014;6:1011-6.

5. Watanabe K, Nomori H, Ohtsuka T, et al. Usefulness and complications of computed tomography-guided lipiodol marking for fluoroscopy-assisted thoracoscopic resection of small pulmonary nodules: experience with 174 nodules. J Thorac Cardiovasc Surg 2006;132:320-4.

6. Ciriaco P, Negri G, Puglisi A, et al. Video-assisted thoracoscopic surgery for pulmonary nodules: rationale 
for preoperative computed tomography-guided hookwire localization. Eur J Cardiothorac Surg 2004;25:429-33.

7. Sugi K, Kaneda Y, Hirasawa K, et al. Radioisotope marking under CT guidance and localization using a handheld gamma probe for small or indistinct pulmonary lesions. Chest 2003;124:155-8.

8. Powell TI, Jangra D, Clifton JC, et al. Peripheral lung nodules: fluoroscopically guided video-assisted thoracoscopic resection after computed tomographyguided localization using platinum microcoils. Ann Surg 2004;240:481-8; discussion 8-9.

9. Rho J, Lee JW, Quan YH, et al. Fluorescent and Iodized Emulsion for Preoperative Localization of Pulmonary Nodules. Ann Surg 2019. [Epub ahead of print]. doi: 10.1097/SLA.0000000000003300.

10. Kuo SW, Tseng YF, Dai KY, et al. Electromagnetic Navigation Bronchoscopy Localization Versus Percutaneous CT-Guided Localization for Lung Resection via Video-Assisted Thoracoscopic Surgery: A PropensityMatched Study. J Clin Med 2019;8. 379.

11. Hyun K, Park IK, Song JW, et al. Electromagnetic navigation bronchoscopic dye marking for localization of small subsolid nodules: Retrospective observational study. Medicine (Baltimore) 2019;98:e14831.

12. Hsu PK, Wu YC. The feasibility of electromagnetic navigation-guided percutaneous microcoil localization for thoracoscopic resection of small pulmonary nodules. J Thorac Cardiovasc Surg 2019;157:e211-e214.

13. Goldstraw P, Chansky K, Crowley J, et al. The IASLC Lung Cancer Staging Project: Proposals for Revision of the TNM Stage Groupings in the Forthcoming (Eighth) Edition of the TNM Classification for Lung Cancer. J Thorac Oncol 2016;11:39-51.

14. Lin JB, Qiu ML, Lin CJ, et al. Simplified synchronous disconnection of pulmonary arteries and veins for right upper lobectomy. Surg Endosc 2019;33:2015-23.

15. Lin JB, Chen JF, Lai FC, et al. Transareolar pulmonary bullectomy for primary spontaneous pneumothorax. $\mathrm{J}$

Cite this article as: Zhou C, Li X, Li W, Qian J. Clock dial integrated positioning combined with single utility port videoassisted thoracoscopic surgery: a new localization method for lung tumors. J Thorac Dis 2021;13(2):1143-1150. doi: 10.21037/ jtd-20-3312
Thorac Cardiovasc Surg 2016;152:999-1005.

16. Nachira D, Meacci E, Porziella V, et al. Learning curve of uniportal video-assisted lobectomy: analysis of 15 -month experience in a single center. J Thorac Dis 2018;10:S3662-S3669.

17. Leone FT, Evers-Casey S, Toll BA, et al. Treatment of tobacco use in lung cancer: Diagnosis and management of lung cancer, 3rd ed: American College of Chest Physicians evidence-based clinical practice guidelines. Chest 2013;143:e61S-e77S.

18. Weimann A, Braga M, Carli F, et al. ESPEN guideline: Clinical nutrition in surgery. Clin Nutr 2017;36:623-50.

19. Ha D, Mazzone PJ, Ries AL, et al. The Utility of Exercise Testing in Patients with Lung Cancer. J Thorac Oncol 2016;11:1397-410.

20. Altorki NK, Kamel MK, Narula N, et al. Anatomical Segmentectomy and Wedge Resections Are Associated with Comparable Outcomes for Patients with Small cT1N0 Non-Small Cell Lung Cancer. J Thorac Oncol 2016;11:1984-92.

21. Cheng X, Zheng D, Li Y, et al. Tumor histology predicts mediastinal nodal status and may be used to guide limited lymphadenectomy in patients with clinical stage I non-small cell lung cancer. J Thorac Cardiovasc Surg 2018;155:2648-2656.e2.

22. Ichinose J, Kohno T, Fujimori S, et al. Efficacy and complications of computed tomography-guided hook wire localization. Ann Thorac Surg 2013;96:1203-8.

23. Lin MW, Tseng YH, Lee YF, et al. Computed tomography-guided patent blue vital dye localization of pulmonary nodules in uniportal thoracoscopy. J Thorac Cardiovasc Surg 2016;152:535-544.e2.

24. Sui X, Zhao H, Yang F, et al. Computed tomography guided microcoil localization for pulmonary small nodules and ground-glass opacity prior to thoracoscopic resection. J Thorac Dis 2015;7:1580-7.

(English Language Editor: A. Kassem) 